\title{
EL ESTUDIO GEOGRÁFICO DE LA ENERGÍA: UNAAPROXIMACIÓN HISTÓRICA AL ESTADO DE LA CUESTIÓN
}

\author{
Daniel Herrero Luque \\ Eugenio Baraja Rodríguez \\ Departamento de Geografía. Universidad de Valladolid \\ danielherreroluque@gmail.com, baraja@fyl.uva.es
}

\section{RESUMEN}

De entre las actividades antrópicas la producción energética es una de las que ha generado mayor impacto espacial y transformación del paisaje. La comunidad de geógrafos, si bien se interesó de forma temprana por los recursos energéticos o su aprovechamiento y consumo, no ha abordado hasta el nuevo siglo la dimensión paisajística de la energía. El artículo analiza a partir de un trabajo de revisión bibliográfica el estado de la cuestión de los estudios geográficos sobre la energía. Los resultados identifican las orientaciones con las que ha sido abordada la "geografía de la energía" y las líneas de investigación presentes.

Palabras clave: Geografía de la energía, estado de la cuestión, energías renovables, recursos energéticos.

\section{ABSTRACT}

Among the anthropic activities, both mining and energy development are uppermost in terms of spatial impact and their capacity for landscape transformation. While geographers took an early interest over energy resources, it was not until the turn of the new century that they approached the landscape dimension of energy. The present paper engages in a literature

Fecha de recepción: octubre 2015.

Fecha de aceptación: enero 2017. 
review in order to assess the current state of geographical studies on the issue of energy. Our results point at both the main past approaches to the study of the so-called "geography of energy" and the current lines of research in this field.

Keywords: Geography of energy, state-of-the-art, renewable energy, energy resources, literature review.

\section{INTRODUCCIÓN}

La geografía siempre se interesó por los recursos naturales y las materias primas, pero es desde hace unos años que los trabajos en dicha materia se han multiplicado. ¿Qué dimensiones han abordado los estudios geográficos sobre la energía y los recursos mineros? ¿Qué lugar ocupa el paisaje en dichos estudios? Hasta hace una década los estudios geográficos que tienen por objeto profundizar en el paisaje de dominante minera y/o energética han sido prácticamente inexistentes. Los estudios sobre la explotación minera o el aprovechamiento de recursos energéticos no consideran el paisaje como objeto de análisis independiente y mucho menos la capacidad de la actividad energética y minera de generar nuevos paisajes. La emergencia de paisajes de dominante energética no ha sido abordada por la geografía española hasta hace una década. Hasta entonces los estudios geográficos únicamente señalaban las consecuencias destructivas que la actividad energética y/o minera producían sobre los paisajes rurales, sin considerar que ésta favorecía la emergencia de nuevos paisajes.

Los estudios sobre los recursos energéticos, su explotación, producción y distribución en el espacio constituyen un tema clásico en la investigación geográfica. Resulta conveniente identificar y revisar los trabajos realizados principalmente por aquellos geógrafos interesados en cuestiones energéticas, donde se proyectan los enfoques y paradigmas propios de cada época. La energía ha sido objeto de amplios estudios por parte de geógrafos. Los primeros que se publican sobre los diferentes recursos energéticos son los de carácter exploratorio, centrados en la localización, potencial de explotación, etc. Pero si focalizamos la atención sobre estudios geográficos más avanzados, o sobre la energía en términos globales, identificamos otras dimensiones, abordando la energía (i) como una actividad económica y por ende generadora de riqueza, (ii) como un modo de transformación y de "agresión" al medio natural, y (iii) como elemento clave que intensifica el debate territorial y despierta la conciencia social sobre el valor del paisaje. Éstas son las tres principales dimensiones que se han elaborado en la medida en que los geógrafos han investigado sobre la energía y sobre las que se profundiza en el presente artículo.

\section{ESTUDIOS GEOGRÁFICOS EXPLORATORIOS SOBRE RECURSOS ENERGÉTICOS}

La energía y los recursos mineros han sido clave en los estudios desarrollados dentro de la geografía de la producción, de los intercambios y del comercio. El interés por parte de los geógrafos se centró en un primer momento en el estudio de las condiciones geológicas y técnicas que permitían la explotación de los recursos; para seguidamente focalizar la atención en la disponibilidad de reservas y las condiciones económicas que permitían la explotación 
de nuevos yacimientos. Ese esquema se reproduce en los trabajos sobre los diferentes recursos minerales y energéticos que fueron abordados por los geógrafos, en primer lugar sobre el carbón, pero posteriormente ampliados al petróleo, gas natural y uranio -energía nuclear-. Esta dimensión productiva o económica está estrechamente vinculada a los primeros estadios de investigación sobre cualquier recurso, a modo de trabajos exploratorios; así como a estudios regionales de carácter general. No conviene establecer unos umbrales temporales, pues en las últimas décadas se han publicado estudios de carácter exploratorio sobre novedosas técnicas de producción energética, destacando trabajos sobre los biocombustibles, la cogeneración, la energía termosolar, etc.

Los estudios geográficos de carácter exploratorio parten de la inexistencia o limitación de estudios previos, por lo que las tareas de recopilación y enumeración de la producción y de los intercambios del recurso, así como de su localización, son necesarias. A las cuestiones de la producción agraria e industrial más tradicionales en geografía se unió pronto la producción de energía, así como su intercambio y distribución. Estos tres aspectos aparecen en la definición de geografía económica propuesta por Allix en 1948, quien define su objeto como "el estudio de la coordinación de hechos de producción, consumo e intercambio" (1948: 297). La geografía económica, como señaló Claval, se consolidó ya en el último cuarto del siglo XIX como lo atestiguan los manuales de geografía comercial. Éstos ofrecen inventarios y registros precisos de recursos por país y tipo de producto. Esta corriente se consolidó a partir de la década de 1920, adquiriendo gran desarrollo en la geografía española y francesa durante las décadas 1950 y 1960 . El principal objeto de estos trabajos era tomar conciencia de las transformaciones del mundo moderno y principalmente de los cambios económicos que acontecían en esos años -especialmente los que afectaban a las sociedades y formas de vida tradicional-. A partir de los años setenta, la dimensión económica evoluciona, con motivo de la primera crisis del petróleo, y la energía se transforma en el elemento clave ante la amenaza de agotamiento de los principales recursos energéticos a corto plazo.

Los recursos energéticos y mineros fueron ampliamente estudiados de forma independiente en numerosas publicaciones. El esquema elaborado en dichos trabajos era eminentemente descriptivo, abarcando desde la génesis del recurso, su localización, características, la historia de su explotación, o el potencial en los diferentes países. Se trata pues de estudios a escala mundial donde el potencial de las distintas áreas de producción era examinado y evaluado sin centrar su atención en los paisajes resultantes de la diversidad de condiciones históricas, técnicas y económicas vinculadas a la extracción minera y/o aprovechamiento energético.

Las publicaciones sobre el carbón son numerosas, pues se trata de un combustible que ha ejercido una fuerte influencia en el desarrollo industrial, así como en la transformación profunda de determinados territorios. Las primeras obras publicadas en los años treinta y cuarenta del siglo XX lo conforman estudios de carácter regional, que describen en primer lugar los aspectos geológicos del área de estudio y realizan un estudio de la evolución histórica de la actividad extractiva. Ya en los años cincuenta la industria y la localización de infraestructuras energéticas adquiere mayor relevancia. A mediados de la década de 1960 se empezó a estudiar los factores que participan en el crecimiento y/o en el declive de la actividad minera. La demanda de grandes cantidades de agua y carbón asociada a las centrales térmicas, su localización, la generación de residuos y los posibles trastornos en el entorno inmediato fueron las cuestiones más estudiadas durante los años setenta y ochenta. 
De forma sucesiva fueron otros recursos los que cobraron mayor relevancia en los trabajos de los geógrafos, repitiendo el mismo esquema que con el carbón -desde la génesis del propio recurso hasta su descubrimiento y posterior aprovechamiento o explotación-. La obra de Pratt y Good de 1950 titulada World geography of petroleum se publicó a través de la Sociedad Geográfica Americana, partiendo de un discurso centrado en los recursos y producciones minero-energéticas. Posteriormente dan un giro e inician una discusión sobre la relación entre recursos y actividad económica. La discusión está centrada en el estudio de la organización y el funcionamiento de la actividad petrolera y el análisis de los diferentes procesos desde su extracción hasta el consumo. A partir de la década de 1970 se incrementaron los trabajos sobre la distribución, la producción, el transporte y el aprovechamiento del petróleo y el gas natural. Dentro de éstos, los estudios regionales adquirieron gran relevancia. Un nutrido número de obras de geografía económica ofrecieron investigaciones sobre el petróleo y el gas natural ${ }^{1}$.

Durante los años sesenta y setenta la energía nuclear iba adquiriendo mayor importancia como un suplemento a las centrales térmicas de generación eléctrica a partir de carbón. A pesar del interés que suscitó en la población, no atrajo la atención de muchos geógrafos como objeto de estudio desde una dimensión económica. Algunos artículos vieron la luz, como el de M. Michel (1978), un ejemplo muy representativo, aunque tardío, de los estudios tradicionales de los años sesenta. Realiza un análisis completo sobre las condiciones de extracción de uranio y examina las características geológicas de los yacimientos, las técnicas de explotación, los principales productores y el aprovechamiento del uranio, así como la organización económica de la producción. Las referencias a cuestiones ambientales o a la propia energía nuclear son muy limitadas desde la geografía, y estarán inscritas en estudios bajo una dimensión netamente ambiental.

Los geógrafos han demostrado ampliamente el interés en los recursos energéticos de naturaleza renovable, en un principio fundamentado en la popularidad que adquirieron por su carácter alternativo a los recursos fósiles. Antes de producirse la expansión espacial de técnicas de aprovechamiento de los actuales recursos renovables -fotovoltaico, eólico, biocombustible, etc.- el agua adquirió gran relevancia. El desarrollo de la energía hidroeléctrica data de finales del siglo XIX. La energía hidroeléctrica es una de las técnicas de generación eléctricas más tradicionales a partir de un recurso renovable (Frolova et al., 2015), lo que justifica su consideración en los múltiples estudios regionales realizados desde la disciplina geográfica. Los recursos energéticos renovables y las nuevas técnicas, como el resto precedentemente han sido objeto de cuantificación, donde la potencia instalada de las centrales y la electricidad vertida a la red se han perfilado como factores que justificaban el desarrollo económico de los territorios en términos económicos y energéticos. También se han hecho interesantes estudios como el de Espejo y García (2010), donde se analiza la evolución de la

1 Uno de los primeros trabajos fue An economic geography of oil, de Odell (1963). Alexander y Gibson (1979) realizaron una revisión histórica de la geografía del petróleo y del gas natural. La geógrafa americana Ira M. Sheskin publicó una revisión bibliográfica al respecto en la obra colectiva Geographical dimensions of energy (1985). De esta última obra extraemos las principales cuestiones que han sido abordadas desde la geografía: El impacto económico y la importancia de la energía en Oriente Medio. Los yacimientos de petróleo y gas natural del Mar del Norte. La red de oleoductos que conecta las centrales de extracción con los centros de consumo. Los problemas específicos en determinados ámbitos: Nigeria, Florida, Alaska, China, California, etc. 
energía hidroeléctrica en España. Más recientemente, la hidroelectricidad aparece estrechamente vinculada a la aceptación y consolidación de paisajes determinados, donde la energía se constituye como un objeto cultural singular (Briffaud et al., 2015). Las crisis petroleras de la década de 1970 impulsaron el auge de "energías alternativas" y con ellas nuevas oportunidades profesionales y una nueva lógica espacial. Un auge y entusiasmo que no se materializó en la medida esperada, interrumpiéndose los estudios iniciados sobre el potencial y la planificación de aprovechamiento, la valoración de los recursos o la política energética. Trascurridas casi dos décadas y gracias el avance tecnológico y el apoyo público de determinadas formas de energía, como la eólica y la fotovoltaica, el panorama energético de numerosos países comenzó a transformarse. Con el cambio de siglo, y partiendo de la inexistencia de estudios continuados previos, se reprodujo el esquema inicial de los trabajos sobre recursos energéticos realizados en décadas pasadas. Los estudios geográficos de carácter exploratorio sobre la energía en las últimas décadas resultan de gran relevancia. Gracias a ellos la comunidad científica y el público en general logra comprender la dimensión y las características de sectores en auge como el de los biocombustibles (Espejo, 2009; 2016) entre otros de naturaleza renovable. Los trabajos de carácter exploratorio ahondan en el potencial energético, la disponibilidad y carácter de los recursos o los agentes involucrados. Se trata de trabajos a los que les acompaña en la mayoría de los casos una ardua tarea de recopilación y enumeración de la producción y de los intercambios del recurso, así como de su localización.

\section{GEOGRAFÍA DE LA ENERGÍA: DISCURSOS INTEGRADORES DENTRO DE LA GEOGRAFÍA ECONÓMICA Y SOCIAL}

Además del tratamiento independiente de los diferentes recursos energéticos, los geógrafos han dedicado un gran esfuerzo al estudio de la energía como categoría, en el sentido más amplio, lo que se ha denominado "geografía de la energía". Ese término, aunque ampliamente usado, no se formula de manera unívoca, y su conceptualización ha concentrado un gran esfuerzo de la comunidad de geógrafos. Para Wilbanks (1985: 505-508), la especificidad de la dimensión geográfica en torno a la energía está vinculada a su sentido espacial y a su proximidad a tres conceptos: localización, estructura y movimiento. Precisamente una obra titulada Géographie de l'Énergie (George, 1950) se convirtió en una referencia clave para entender el desarrollo que adquirió el estudio geográfico de la energía. Pierre George publicó su obra dentro de la colección de geografía económica y social ${ }^{2}$ compuesta por cuatro tomos. El contenido de la obra excede los límites del conocimiento sobre la energía desde la geografía en el momento, destacando la relevancia de los medios de producción en la organización del espacio. Se trata de una temprana y sólida reflexión marxista ${ }^{3}$ que rompe con los estudios físicos y rurales tan predominantes en la geografía francesa.

2 Los otros volúmenes eran Régions, nations, grands espaces de P. Claval, Géographie agraire de D. Fauchery y Géographie de la circulation de R. Clozier.

3 Horacio Capel afirma que ello se debe en gran parte a "la activa participación de los comunistas en la resistencia contra los alemanes durante la Segunda Guerra Mundial" (2012: 371), lo que permitió que "el partido comunista -que llegó a estar en el gobierno tras la Liberación-gozara de un prestigio político e intelectual que no tenía en otros países occidentales en la misma época" (2012: 371). Ello posibilitó por lo tanto un fecundo desarrollo de la ciencia social francesa en los años sesenta. 
En Géographie de l'Énergie P. George realiza una lectura sobre las principales fuentes energéticas presentes en el planeta: el carbón, el petróleo y la electricidad, mostrando el método que le va a caracterizar: un inventario sistemático, así como una evaluación cuantitativa de los yacimientos de energía fósil. A ese trabajo le añade una descripción de las condiciones geológicas de su explotación, acompañada de un análisis de los sistemas de explotación tanto capitalista como socialista. Todo ello lo presenta debidamente justificado a partir de tablas, gráficos y mapas donde muestra la localización de los yacimientos a pequeña escala.

Al finalizar, Pierre George puntualiza que "el objeto de esta obra no ha sido simplemente ofrecer a los estudiosos y al público en general un informe sobre la producción del carbón, del petróleo o de la electricidad. Esto sería sin duda una obra útil, pero que pasaría rápidamente de actualidad por la acción productiva de los hombres. Nuestro esfuerzo principal se ha dirigido al estudio de las condiciones de explotación, y principalmente al estudio de las formas de utilización" (George, 1950: 401). Acompañado en alguna ocasión de fotografías de paisajes mineros de Donéts (Figura 1), de Silesia, de Nord-Pas de Calais y de las explotaciones petrolíferas estadounidenses, Pierre George no hace ninguna mención especial a los paisajes. Éstos permanecen ocultos tras los comentarios de tablas de estadística sobre la producción de carbón por zona y año, las inversiones realizadas por las sociedades mineras, los ingresos de cada explotación minera y el consumo medio de electricidad por habitante. Todos esos datos son considerados por P. George como un indicador de desarrollo ${ }^{4}$. Géographie de l'Énergie ha de ser entendida, pues, como una referencia bibliográfica que estableció el inicio del estudio de le energía desde la dimensión propiamente geográfica ${ }^{5}$-social y económica-.

Los autores más críticos con la obra de Pierre George respondieron con otros trabajos donde pretendieron ser completamente objetivos y evitar en sus investigaciones los juicios de valor u otras posturas políticas o morales. Sin embargo, pocos años después, Jean Piaget puso de manifiesto la dificultad para alcanzar una objetividad en las ciencias del hombre, ya que el hombre es en ellas a la vez sujeto y objeto de conocimiento (Piaget, Lazarsfeld y Mackenzie, 1975: 75). El caso es que quizás por el carácter subjetivo de la publicación de George o por la proximidad a la geografía social, su obra inició un denso debate: ¿Qué es la geografía de la energía? ¿Qué cuestiones aborda? Las obras que sucedieron a esa primera Geografía de la Energía (Géographie de l'Énergie) de P. George mostraron de manera convincente cómo la geografía y la energía pueden resultar interesantes dentro de un mismo estudio. Cada obra centra su atención sobre cuestiones diferentes como el transporte, la logística, el suministro, la localización, la demanda, así como los mercados y la política.

4 De ese modo, en la introducción él explica que "es posible definir el grado de evolución económica de un país por el cociente individual de disponibilidad energética [...] Un Estado que consume poca energía es un país de economía atrasada y, por el mismo hecho, subordinada" (George, 1950, p. 7). Pierre George muestra la superioridad del sistema socialista, y lo hace a partir del estudio de resultados y hazañas de la producción industrial y minera.

5 Los trabajos de Pierre George han sido citados como obras representativas dentro del amplio espectro de la geografía económica y social, con evidentes aportes marxistas. Géographie de l'Énergie tan pronto es considerada como el primer trabajo de geografía económica de la energía (Chapman 1989, 16; Pasqualetti 2011, 973), como una obra anquilosada en planteamientos descriptivos (Manners 1964; Guyol 1971). Ninguno de los dos se contradicen, pero sí reflejan la diferente consideración que los geógrafos han otorgado a Géographie de l'Énergie. 


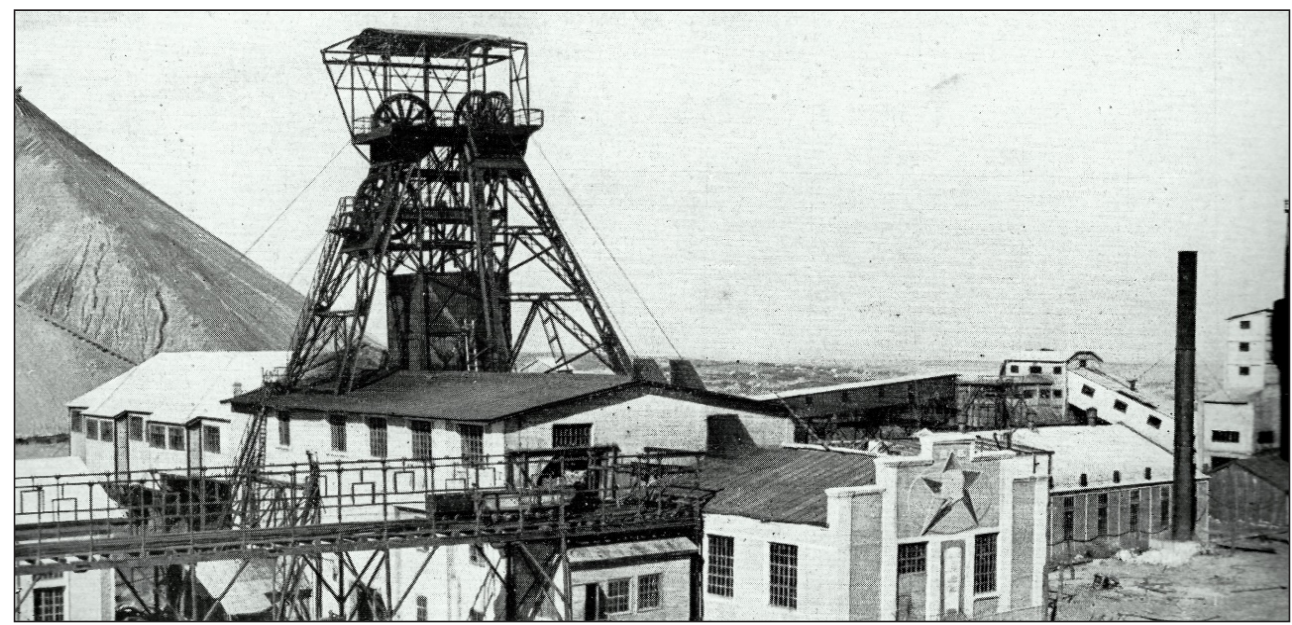

Fuente: Géographie de l'Énergie de P. George (1950, lámina 2). Fuente original : Oficina de Información Soviética.

Como resultado, las obras de energía proliferan en la comunidad de geógrafos. Se trata de investigaciones realizadas en el seno de la geografía económica, asentada bajo unos preceptos positivistas y teoréticos, apoyada en técnicas y métodos cuantitativos de análisis. El esquema que seguían estos trabajos, propio de la geografía económica -que tan buena acogida tuvo de forma especial en la geografía francesa y española- se basaba en la disponibilidad de recursos, las reservas explotables y la producción energética. Una geografía que hizo que se ganara su reputación de disciplina enciclopédica donde el ser humano, así como los paisajes, apenas tenía cabida junto a las tablas estadísticas. El resultado de numerosos trabajos se reducía a un inventario sistemático y preciso, donde primaba la preocupación por la producción y la enumeración de productos y producciones ${ }^{6}$. Los productores y las relaciones existentes entre ellos serán los grandes ausentes. "En este contexto las cifras sobre recursos económicos, producción y productos, población y comercio, eran nociones y conceptos abstractos, más o menos aislados de la estructura económica, de las técnicas de producción y de las relaciones sociales" (Dresch, 1980). Las teorías de localización sustentaron numerosos estudios, dentro de esa base conceptual y teórica de la economía neoclásica.

Unos buenos exponentes de la dimensión económica que adquiere la energía en la disciplina geográfica son: The geography of energy de Gerald Manners (1964), Energy in the Perspective of Geography de Nathaniel Guyol (1971), Géographie mondiale de l>énergie de Donald W. Curran (1973), Man, Energy, Society de Earl Cook (1976), La nouvelle donne énergétique publicado por D.W. Curran (1981).

6 Las investigaciones sobre los recursos energéticos se centraron en cuatro cuestiones: la formación, la localización, la explotación y los cálculos y valoraciones generales de los recursos. 
El libro The geography of energy de Gerald Manners (1964) consolida el estudio de la energía como línea de investigación dentro de la geografía económica. Gerald Manners critica a aquellos geógrafos que pretendieron buscar mediante la descripción y el análisis de la actividad energética, el carácter variable de la vida económica y los intercambios espaciales derivados en diferentes ámbitos. Por ello, él pretende abordar en su estudio de la geografía de la energía los siguientes tres conjuntos de preguntas que abordan las siguientes cuestiones (Manners, 1964: 19):

- Las instalaciones energéticas: ¿dónde se localizan, por qué se localizan ahí y cómo varían sus productos de salida de un lugar a otro en cuanto a cantidad y a calidad? ¿Presenta patrones estacionales? En caso de fluctuar, ¿Cuál es su tendencia?

- El transporte de la energía. ¿Desde dónde y hacia dónde es transportada la energía y por qué? ¿Cuáles son los aspectos económicos y métodos de tal transporte?

- El consumo de energía. ¿Qué cambios están teniendo lugar en los patrones geográficos del consumo de energía? ¿Cuál es el mix energético de una determinada región o economía? ¿Está cambiando con el tiempo? Los patrones de demanda presentan variaciones temporales y espaciales ¿Cuáles son las repercusiones?

Para el geógrafo inglés, la geografía de la energía está apoyada en los estudios sobre las características espaciales de la producción, transporte y consumo de energía. Coincide con P. George en considerar la distribución de los recursos energéticos como elemento rector de lo que denominan "geografía de la energía". Pero a su vez, Manners detalla otros elementos que considera claves para abordar el tema desde la dimensión geográfica: mercados energéticos, el transporte de energía y factores políticos. Otro aspecto al que se enfrenta el lector ante la obra de Manners y que difiere de las obras francesas previas (Sorre, 1948; George, 1950) es que solo considera aquellas fuentes energéticas de carácter inanimado, suministradas a través de máquinas. Por lo tanto, la dimensión histórica y la relevancia de la energía solar y de la alimentación como fuentes clave permanecen relegadas a la comunidad de geógrafos franceses.

La obra de Manners logró consolidar la cuestión energética en el seno de las investigaciones de la comunidad geográfica y en concreto de la geografía económica. Además, logró centrar la atención de los estudios hacia los costes emanados de la explotación de las materias primas, de producción de energía y de la mano de obra; a los que se sumaron los costes de transporte, el flujo de energía y la accesibilidad de los mercados. Nuevas consideraciones que se extendieron entre los geógrafos anglosajones ${ }^{7}$, como se percibe en la obra titulada Energy in the perspective of geography, publicada en 1971 por Nathaniel Guyol ${ }^{8}$.

7 En cambio los autores franceses continuaron con estudios que tendían más hacia la clasificación y hacia el estudio de evoluciones como la obra de Donald W. Curran Géographie mondiale de l'énergie (Curran, 1973). En España se siguió la tradición francesa en lo que a publicaciones sobre recursos energéticos se refiere, puesto que nunca se publicó un libro donde se abordaran las cuestiones energéticas en un sentido amplio y escala mundial.

8 El autor - geógrafo y economista- trabajó en el departamento de economía de Standard Oil Company, donde adoptó un método cuantitativo novedoso para la fecha, del que se sirve en esta publicación 
Figura 2

IMAGEN EXTRAÍDA DEL LIBRO THE GEOGRAPHY OF ENERGY SOBRE LOS FLUJOS ENERGÉTICOS DE LA JUNTA CENTRAL DE PRODUCCIÓN DE ENERGÍA ELÉCTRICA ENTRE 1956 Y 1966

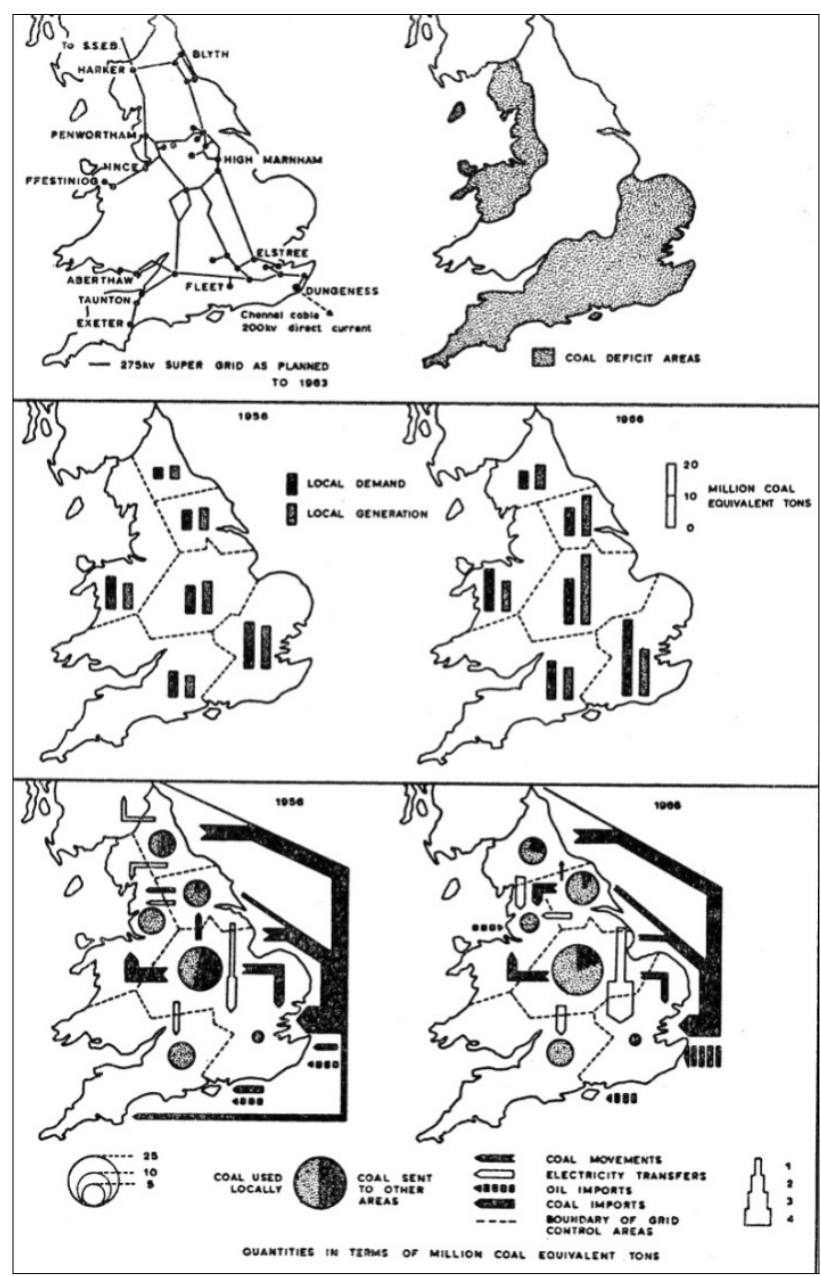

Fuente: The geography of energy, Manners, 1964: 113.

La obra de Guyol se ubica dentro de la serie de volúmenes editados por Foundations of Economic Geography Serie, que pretende explorar en profundidad cuestiones de gran relevancia e interés dentro de la geografía económica, partiendo de profusos análisis cuantitativos -la población, los centros comerciales, el comercio, la venta al por mayor...-. Energy in the perspective of geography, expone con mayor carácter internacional que la obra de Manners (1964) cuestiones relativas al consumo y la demanda de energía, partiendo de densos datos estadísticos. Precisamente es la labor de compilación, de análisis y de síntesis de los datos, lo que otorga a esta obra un merecido reconocimiento. El autor justifica dicho 
tratamiento estadístico en el escaso conocimiento previo sobre: cuánta influencia ejercen los factores que afectan a la demanda de energía; las cantidades de energía usada o la elección de fuentes energéticas (Guyol, 1971: ix). No obstante, los datos ofrecidos por Guyol y las cuestiones que abordó -como fueron la oferta y la demanda energética (Figura 3), o los factores que afectan a la demanda de diferentes sectores económicos-, no vaticinaron el incremento de precios del crudo que agitaron pocos años después la economía mundial.

Figura 3

PRODUCCIÓN Y CONSUMO MUNDIAL DE LOS RECURSOS ENERGÉTICOS COMERCIABLES EN 1965

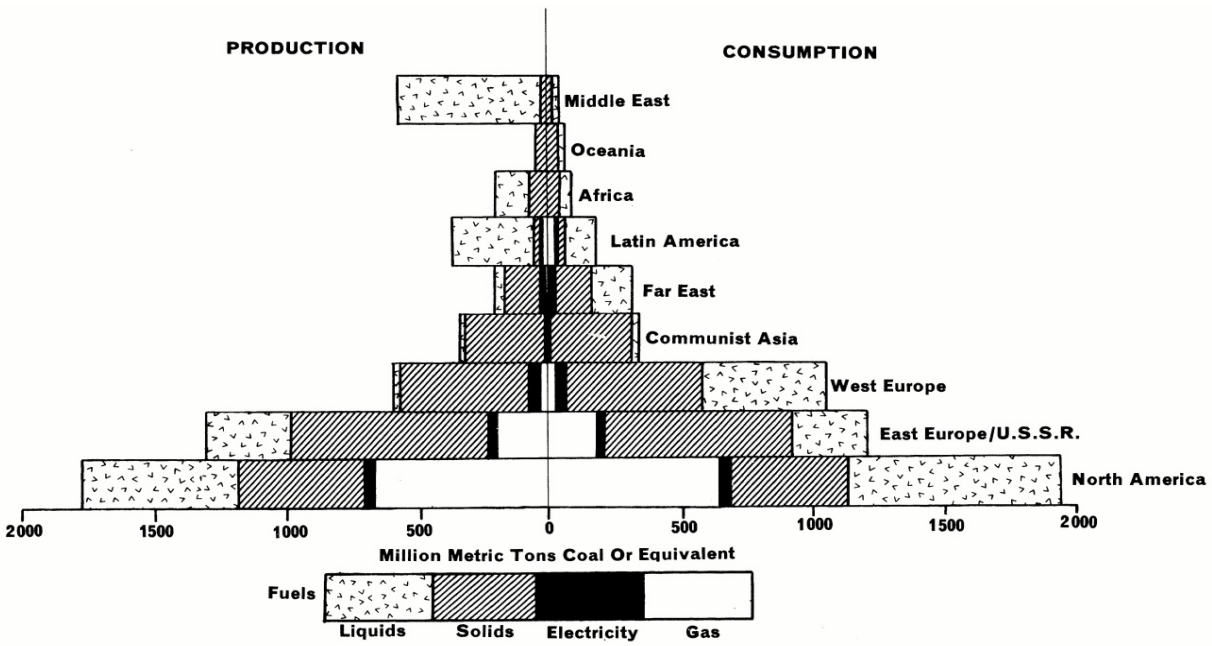

Fuente: Energy in the perspective of geography de N. Guyol (1971: 16)

Lo cierto es que en las mismas fechas en las que la obra de N. Guyol se publicaba, el Club de Roma auguraba el agotamiento de los recursos petroleros, así como los de cobre y plomo, para el horizonte 1990-2000, un panorama alarmante que justificó la preocupación de sus miembros por mejorar el futuro a largo plazo (Turner, 2008: 400), así como de la comunidad científica. Los métodos cuantitativos empleados en los estudios geográficos centrados en la extracción de recursos energéticos o en la producción energética la geografía de la década anterior, tuvieron gran acogida dentro de este panorama alarmista, justificado a partir de tablas estadísticas y de razonamientos a escala planetaria, alejados del terreno y de las sociedades locales. En las décadas de 1970 y 1980 las comparaciones de la extracción de carbón por habitante entre los países socialistas y capitalistas quedan atrás, desarrollándose trabajos sobre cálculos de consumo de carbón o de petróleo por habitante y las estimaciones sobre la duración de las reservas ${ }^{9}$.

9 Cabe señalar el interés actual de procesos que acontecieron en esos momentos, de estudios que partían del crecimiento demográfico de los países del tercer mundo y del incremento del consumo en ciertos países en proceso de industrialización. En concreto destaca el reciente crecimiento de China, que absorbe cantidades enormes de materias primas y de energía, y donde podemos establecer uno de los factores del fuerte incremento del precio del petróleo y del carbón. 
A partir de la alarma generada por el incremento de los precios del petróleo en 1973, la energía emergió como una cuestión que suscitó gran interés en la comunidad científica y en la sociedad. Para los geógrafos, el incremento de los precios del petróleo y el replanteamiento del suministro energético permanecerán en la base de múltiples estudios ${ }^{10}$. Los geógrafos se adscribieron pues al análisis del panorama empresarial y de las políticas energéticas, así como al estudio del funcionamiento de los mercados. Una obra que merece destacar, y donde la crisis energética ejerció de elemento rector es la del geólogo Earl Cook, titulada Man, Energy, Society. La crisis energética era entendida por el autor como una "rápida disminución de recursos no renovables" (Cook 1976, ix) .El autor plantea unas cuestiones, que no son nuevas pero sí alarmantes: (i) la dependencia de grandes contingentes de población de combustibles fósiles, señalando la imposibilidad de renovación o de reciclado; (ii) la desigual distribución de los recursos fósiles y un fuerte desequilibro frente a los lugares de consumo; (iii) el crecimiento de la población amenaza al equilibrio productivo energético y alimenticio; (iv) la acumulación de residuos energéticos de combustibles fósiles y nucleares.

Earl Cook, aunque de formación era geólogo, aboga por ofrecer al lector "una visión geográfica de la energía que ofrezca los antecedentes necesarios para comprender los problemas contemporáneos" (1976: xi). El interés mostrado por las consideraciones espaciales y sociales de la crisis energética, denotan una orientación diferente respecto a los postulados neopositivistas. En un intento de superar las afirmaciones deterministas que aseguraban que el bienestar social radica en el crecimiento económico, Cook señala que el incremento del consumo de energía y de determinados materiales, genera otras consecuencias de "elevado coste como son: los conflictos armados, la contaminación o la esclavitud económica" (Cook, 1976: 193). Las técnicas de la localización y de la clasificación son comunes en la obra, donde el recurso energético es equiparable a la sociedad en el grado de estudio. Precisamente ésa es la aportación novedosa y destacada respecto a obras precedentes, como expresamente lo revela su título: Man, Energy, Society.

A lo largo de la obra el autor hace referencia de forma constante al agotamiento de los recursos energéticos -entre los que incluye las tierras cultivables y la producción alimenticiay al crecimiento socioeconómico en determinadas áreas. Cook afirma que parece improbable que el estado de crecimiento del que disfrutan los países desarrollados -con todos sus mecanismos económicos y racionalizaciones filosóficas-, pueda sobrevivir a la disminución del aprovechamiento de los combustibles no renovables (Cook, 1976: 203-205). Afirmaciones similares nos permiten identificar un planteamiento de clara inspiración malthusiana, apoyado en la intensificación de la crisis ante la rápida disminución de recursos no renovables. El geógrafo logra así establecer un planteamiento cercano al realizado pocos años antes por el Club de Roma, en Los límites del crecimiento (Meadows et al., 1972). En cambio, no es hasta el capítulo 13 cuando platea el debate entre los presupuestos neomalthusianos y la doctrina optimista del crecimiento económico iniciada por Adam Smith. El autor dice no aportar

10 Fitzmmons y Walton (1978) y Pasqualetti (1979). La nouvelle donne énergétique publicado por D.W. Curran (1981) nos ofrece un buen análisis de los cambios sucedidos en la década de 1970 y un estudio de los "grandes espacios energéticos" -EE.UU., Europa, Japón, OPEP y CAEM-. Como resultado de ello, algunos autores afirman que durante los inicios de la crisis energética y la década de 1970 en general tiene lugar una "orgía de referencias relacionadas con la energía, la mayoría de las veces, realizadas a modo de incursiones emprendedoras con la mera intención de captar el interés del público" (Marcus, 1978: 572). 
nada nuevo al respecto, ni argumentar una de las dos, sino que será "un argumento basado en su propia tendencia a favor del empleo del conocimiento geológico para la interpretación de recursos geológicos, y de historia como una guía para el futuro" (Cook, 1976: 384). Las primeras, las interpretaciones de los recursos geológicos están claramente definidas por su profesor y mentor M. King Hubbert, quien formuló en 1956 la teoría del pico, comúnmente conocida como Peak Oil. Hubbert estimó que para el año 2000 se alcanzarían las cifras máximas de producción mundial de petróleo, declinando posteriormente a un ritmo acelerado. La teoría ha sido ampliamente aceptada por la comunidad científica, así como la industria petrolera, en la que el geofísico americano trabajó.

La obra de Cook dentro del presente estado de la cuestión merece ser destacada al ofrecernos una definición de forma clara y explícita sobre la geografía de la energía. "La geografía de la energía es el estudio de la distribución global de recursos energéticos, de la producción, distribución y utilización de tales recursos, y de las estructuras organizativas o institucionales para aprovechar los recursos" (Cook, 1976: 225). Esta definición viene a dar continuidad y a completar las ofrecidas por anteriores autores como Manners, quien afirma que los trabajos de geografía de la energía han de basarse en el estudio de las "características espaciales de la producción, del transporte y del consumo energéticas" (Manners, 1964: 20). Sin embargo, ninguno de los dos hace mención expresa al carácter transformador de la energía, como sí lo hace Guyol en la introducción de su libro, indicando que la energía desde la perspectiva de la geografía "puede ser definida como la capacidad para modificar las relaciones entre el hombre y su entorno" (Guyol, 1971: ix). Esta afirmación nos muestra una nueva forma de abordar el estudio de los recursos energéticos, y de forma más general de los recursos naturales, de los cuales existe ahora una mayor sensibilidad y comprensión espacial y ambiental.

\section{DIMENSIONES AMBIENTAL Y ESPACIAL DE LOS ESTUDIOS GEOGRÁFICOS SOBRE LA ENERGÍA}

El estudio del impacto de las actividades mineras y de producción de energía sobre el medio tiene antecedentes anglosajones ya en la segunda década del siglo XX. G. K. Gilbert (1917) y R.L. Sherlock (1922) se encargaron de medir los impactos de las explotaciones mineras en las dinámicas fluviales. Evidentemente estos estudios se centraban en regiones de dilatada tradición minera como Inglaterra. En ese mismo tiempo los geógrafos interesados en el impacto de las actividades humanas sobre el medio centraban su atención en la actividad agraria. Para ellos, ésta era el principal factor que justificaba de la progresiva erosión del suelo, como consecuencia de la destrucción de la cobertera vegetal preexistente a la actividad agraria. Las actividades mineras e infraestructuras energéticas existentes hasta mediados del siglo XX generaban un impacto espacial limitado, considerado más escaso en comparación a la actividad agraria. Sin embargo, durante la segunda mitad del siglo XX la actividad minero-energética cambió de escala, lo que contribuyó al incremento del interés de la comunidad geográfica hacia el impacto ambiental y espacial. Inicialmente los geógrafos interesados en la dimensión ambiental analizaron los flujos de energía en los diferentes espacios, configurando "sistemas energéticos" diferenciados. Es el caso de Hare $(1953 ; 1965)$, Thornthwite (1961) y Linton (1965), quienes formaron una masa crítica previa a la vorágine 
de publicaciones desde la geografía económica, más cercanos a variables físicas. Se suceden varias décadas donde la geografía de la energía es una cuestión abordada eminente y repetitivamente desde la geografía económica y social.

Evidentemente surgieron voces discordantes que afirmaban que la geografía de la energía conocida hasta entonces, no poseía un dominio de investigación con carácter propio ni contribuía a la formulación de políticas públicas (Calzonetti y Solomon, 1985: 1). Valga como ejemplo del elevado grado de especialización adquirido en la disciplina geográfica alguna de las críticas que Calzonetti y Solomon recogieron en Geographical dimensions of energy: “con vistas al mercado de trabajo [...] más que geógrafos, salen de las facultades especialistas en sedimentología, hidrografía, en geografía del transporte, de las comunicaciones o de la industria por citar solo algunos" (1985: 1). En respuesta a ello, Wilbanks destaca en la misma obra la importancia y la misión de la Geografía y de los geógrafos en el estudio de la energía. Wilbanks afirma que la energía ha emergido "como un importante tema de especialización en geografía, ofreciendo a los geógrafos la oportunidad para participar en la toma de decisiones durante el proceso de transición energética hacia fuentes no fósiles" (Wilbanks, 1985: 497). En efecto, se produjo una reorientación en el estudio de la energía en la disciplina geográfica, que abogaba la aproximación al terreno y a las comunidades locales ${ }^{11}$.

A finales de la década de los ochenta John Doneric Chapman (1989) logra consolidar una nueva dimensión de los estudios de energía en la comunidad geográfica. Dejó atrás esquemas descriptivos y métodos cuantitativos y profundizó en la noción de sistema energético -iniciado por los geógrafos físicos en la primera mitad del siglo XX y en consonancia con la teoría general de sistemas, ampliamente desarrollada en la disciplina geográfica-. La obra titulada Geography and Energy destaca por el estudio del complejo sistema comercial de la energía y la proximidad a la geografía radical. En la obra cobran relevancia los problemas regionales y locales y los desequilibrios a escala global. Además, Chapman parece haber seguido los consejos de Wilbanks y profundiza en conocimientos sobre energía anteriormente obviados y aborda la planificación energética que permiten al geógrafo participar en la política pública.

Chapman, a la hora de dar un título a su obra renuncia a la preposición de posesión y artículo "de la" -of-, por la conjunción "y" -and- entre geografía y energía. Con Geography and energy el autor establece una relación de igualdad entre los dos términos. Aunque reconozca el elevado grado de especialización que adquiere la energía desde diferentes disciplinas - ciencias básicas, ciencias aplicadas, ciencias ambientales o ciencias sociales y políticas-, Chapman afirma que ello requiere una aproximación interdisciplinar. Asistimos entonces, con el cambio de década, a un pausado pero interesante cambio de planteamiento hacia discursos radicales a partir de consideraciones espaciales y sociales. El crecimiento desigual y la degradación ambiental a la que hicieron referencia numerosos autores radicales de forma recurrente, conforman la base de una nueva dimensión, la espacial y ambiental ${ }^{12}$.

11 En contraposición a los métodos cuantitativos empleados en la geografía productivista de la década anterior, estructurados a partir de tablas estadísticas y de razonamientos a escala planetaria, alejados del terreno y de las sociedades locales.

12 Como antecedentes destacamos algunos geógrafos, principalmente anglófonos, que estudiaron el impacto de la actividad energética en el medio ambiente. Identificamos obras tempranas que se encargaron de medir determinados cambios que la actividad minera generaba en el entorno. Como se ha indicado, G. K. Gilbert (1917) mostró el proceso de transformación del caudal de los ríos como consecuencia de la explotación minera en la Sierra Nevada 
Con el inicio de la década de los noventa, la energía perdió protagonismo en publicaciones y eventos científicos de la comunidad geográfica (Brücher, 1997; 2004), llamativamente cuando en otras disciplinas, como la historia, esta cuestión suscitaba un creciente interés ${ }^{13}$.

Figura 4

PLATAFORMAS PARA LA EXTRACCIÓN DE BITUMEN EN COLD LAKE, ALBERTA, CANADÁ

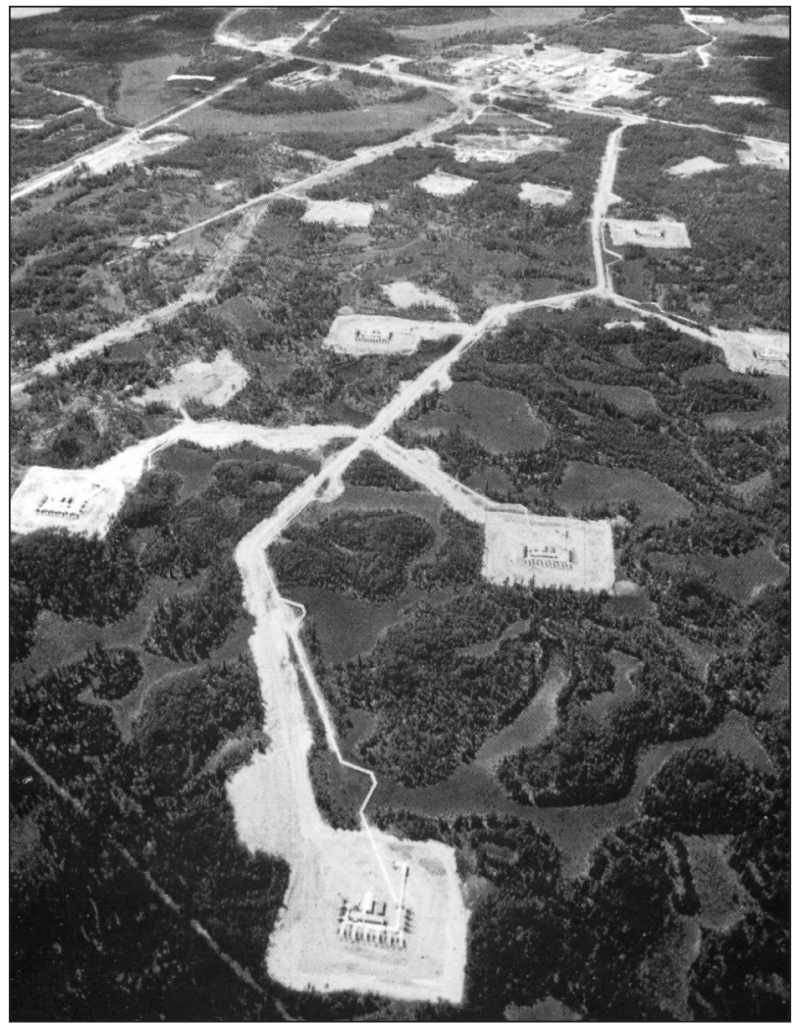

Fuente: Esso Resources en Geography and Energy de J.D. Chapman (1989, lámina 6).

La atonía mostrada a través de los estudios y trabajos sobre la energía desde una óptica geográfica se vio alterada en la primera década del siglo XXI. La energía toma fuerzas en el debate científico global y los geógrafos muestran gran interés. La energía es abordada

californiana. Mientras, R.L. Sherlock (1922) se esforzó en calcular los volúmenes de material desplazado por las actividades extractivas en Gran Bretaña desde sus orígenes. En todo caso, los estudios relativos al impacto de las actividades humanas en el medio tuvieron un desarrollo muy limitado. El principal motivo es que la mayor parte de los geógrafos que se interesaban en esas cuestiones, concentraron su actividad investigadora en la actividad agrícola, concebida como la principal actividad transformadora del medio.

13 Destacan las publicaciones del profesor de historia David E. Nye por su cercanía a la disciplina de Geografía, como es el caso de Electrifying America, Technologies of Landscape, y Consuming Power (Nye 1990). La realización de esos trabajos y otros por historiadores aporta nuevos conocimientos, lo que podemos valorar como aspecto positivo. 
como parte de los estudios genéricos de urbanismo, del transporte y las comunicaciones, el clima, la gestión de los recursos naturales y el desarrollo sostenible. Según MérenneSchoumaker esta variedad de temas donde la energía es abordada muestra la importancia del tema, resultando especialmente interesante en la "comprensión de estructuras espaciales, de dinámicas, de intercambios y en definitiva del mundo en el que vivimos" (2011: 23). La dimensión ambiental, como el resto que estamos identificando en los estudios geográficos sobre la energía, no presentan un umbral cronológico que nos conduzca a hablar de períodos. Ello lo evidencian publicaciones recientes como Routledge Research Companion to Energy Geographies (Bouzarovski et al., 2016). En ella destaca la importancia de la energía dentro del conjunto de aspectos en los que la geografía centra su atención, desde el cambio climático hasta la calidad de vida de las sociedades actuales. La publicación ofrece una perspectiva integrada de las complejas interdependencias entre geografía y energía, abarcando materias económicas, políticas, ambientales, de gobernanza o seguridad entre otras. La energía se perfila como un elemento estratégico y gran reto a escala mundial. La dependencia de los hidrocarburos, la reducción de la emisión de gases de efecto invernadero, las contradicciones en materia de seguridad nuclear o el deseo de consolidar una transición energética hacia un modelo hipocarbónico han favorecido el apoyo público, los avances técnicos y desarrollo comercial de las energías renovables. Estas últimas han centrado la atención de numerosos agentes, entre los que destaca de la opinión pública, el sector industrial y financiero, los organismos internacionales y los poderes públicos. La comunidad de geógrafos tomó conciencia de ello tempranamente, y como resultado se han forjado numerosos foros y proyectos nacionales e internacionales. Por consiguiente las energías renovables, el paisaje y el territorio articulan en la actualidad los estudios geográficos sobre la energía.

\section{ENERGÍAS RENOVABLES Y PAISAJE: NUEVO PARADIGMA DE LA GEOGRAFÍA DE LA ENERGÍA}

El paisaje que resulta de la explotación de recursos energéticos y su transformación apenas ha sido abordado hasta hace dos décadas. Ello se debe no solo al tardío y débil desarrollo industrial -principal actividad demandante de recursos minero-energéticos-, sino fundamentalmente a la concepción propia que del paisaje ha tenido la comunidad geográfica francesa o española, centrada en los paisajes rurales y los estudios regionales. Con el cambio de siglo, la Transición Energética por un lado, y su inmediato producto, las energías renovables (eólica y solar fotovoltaica principalmente), junto a un creciente interés social por el paisaje, han propiciado que se haya colocado el foco en esa relación. Así, numerosos estudios tienen por objeto el análisis de la integración de las energías renovables en el paisaje y la concepción de nuevas lógicas territoriales. El auge y expansión de la energía renovable en Europa y Norteamérica desde finales de la década de 1990 y en otros espacios como América Latina o Asia en la última década, ha evidenciado fuertes vínculos entre la energía, el territorio y el paisaje. La dimensión territorial y paisajística cobra en la actualidad especial relevancia en los estudios abordados desde ciencias sociales y humanas y especialmente desde geografía. Una aproximación de las aportaciones de la comunidad científica sobre la cuestión nos ofrece las claves sobre la relación energía, territorio y paisaje. 
Entendido el paisaje como "cualquier parte del territorio tal como la percibe la población, cuyo carácter sea el resultado de la acción y la interacción de factores naturales y/o humanos" (Convenio Europeo del Paisaje Art.1), consideramos que la percepción ciudadana se erige como un elemento clave en el binomio energía-paisaje ${ }^{14}$, perfilando una relación muy controvertida entre ambos conceptos. Inicialmente la percepción ciudadana cobró especial relevancia en los estudios que focalizan su atención en la población (Walker, 1995) que reside en las inmediaciones de las plantas de producción energética de origen renovable. Numerosos trabajos ahondan en factores difícilmente cuantificables, como los culturales y sociales, así como otros de orden demográfico y económico a partir de estudios de caso (Hammarlund et al., 2016; Baraja y Herrero, 2010; Frolova et al., 2015). El principal objetivo de estos trabajos es conocer el contexto local que condiciona la formación de la opinión. Los resultados de la mayoría de estos trabajos hacen hincapié en aquellos factores que generan conflictos y en especial rechazo, para así identificar los elementos que han de ser valorados en el proceso de toma de decisiones.

La relación controvertida entre energías renovables y paisaje se hace evidente en el momento en que al tiempo que son acogidas de forma positiva -energías limpias, verdes, sostenibilidad, etc.- generan una fuerte oposición. La expansión espacial de las energías renovables, el incremento de su capacidad de producción y tamaño de las infraestructuras y el mayor grado de concentración espacial, han generado la contestación y rechazo de numerosos agentes sociales. Los movimientos de oposición centran su discurso en la localización espacial de las infraestructuras energética y en el proceso de planificación que ha derivado en numerosos conflictos y debates. Con el inicio del siglo XXI emergieron numerosos trabajos de sociólogos y psicólogos sobre la percepción y la aceptación social de las energías renovables (Woods, 2003; Devine-Wright y Devine-Wright, 2006; Haggett y Toke, 2006). Estas aportaciones lograron demostrar la naturaleza de las posiciones de los agentes sociales -apoyo, rechazo y pasividad- frente a los proyectos específicos. Las posiciones o aptitudes de los diferentes grupos sociales no dependen únicamente de la ausencia de sensibilidad hacia los beneficios ambientales de la energía renovable, del escepticismo sobre la tecnología o de la ubicación de proyectos concretos, sino que reflejan valores más profundos, contextos culturales e institucionales más amplios, y reivindican la objetividad y la verdad (Prados et al., 2012: 131). Dichos estudios iniciales de sociólogos y psicólogos permiten contextualizar la dimensión que adquieren los estudios geográficos, centrando su antención en las energías renovables como agentes de conflicto.

Los estudios científicos realizados sobre la capacidad de las energías renovables de generar conflictos son numerosos. Efectivamente, el carácter descentralizado y la expansión de las plantas de energía han posibilitado profusos análisis de los impactos originados. Prados et al., (2012) identifican dos discursos que señalan a las energías renovables como agentes de conflicto (Prados et al., 2012). El primero vinculado a la ordenación del territorio y la planificación energética. Zoellner et al (2008: 4140) afirma que el desarrollo de proyectos de energías renovables socialmente aceptados debe vincular ambos elementos: planificación

14 La participación ciudadana y la percepción social y los valores paisajísticos han sido dos factores del desarrollo eólico en España identificados por Frolova (2010b) a partir de sus investigaciones y otros autores (Devine-Wright and Devine-Wright, 2006; Wolsink, 2007; Wüstenhagen, Wolsink y Bürer, 2007; Toke, Breukers y Wolsink, 2008). 
energética y territorial. Un principio que parece básico, pero en el que subyace el interés de encontrar localizaciones idóneas tanto en términos de disponibilidad del recurso energético, como de una menor afección a la calidad del paisaje (Díaz y Domínguez, 2015). Este discurso aboga pues por la alianza de territorio y energía en materia de planificación, tanto en espacios protegidos (Afonso y Mendes, 2015; Deshaies y Herrero, 2015) como el resto, con el fin de establecer un marco de referencia en la inserción física de las centrales de producción energética en el paisaje. El segundo discurso, según Prados et al., (2012: 133) centra su atención de forma específica en los "paisajes de las energías renovables" dominados por el impacto visual y la visión estática del paisaje. No obstante recientemente se están priorizando aspectos como la protección, ordenación y gestión de los paisajes y otros de gobernanza territorial. Algunos autores (Cowell, 2010; West, Bailey y Winter, 2010 en Prados et al., 2012: 133) añaden que en este discurso está presente el paisaje en tanto aglutinador de cualidades ambientales, culturales y territoriales (Baraja et al., 2015; de Andrés et al., 2015), que deben ser incorporadas a las estrategias de planificación. El objetivo final y práctico de los trabajos apoyados en este discurso es el de identificar localizaciones idóneas y así evitar que las instalaciones de energía renovables contribuyan a la degradación del paisaje (Möller, 2006; 2010; Nadaï y Labussière, 2010; Nadaï et al., 2010; van der Horst y Toke, 2010).

Los geógrafos a menudo han impuesto una visión restrictiva del paisaje, a modo de manifestación perpetua de la adaptación de los grupos humanos a un medio particular. De ese modo la actividad de extracción de los recursos energéticos y la producción de energía provocan una ruptura entre las sociedades y el espacio natural, lo que es percibido como una fuerza de destrucción del paisaje, o como una fase transitoria. Los aerogeneradores y módulos fotovoltaicos salpican el paisaje desde hace más de dos décadas, y numerosos estudios geográficos sobre las energías renovables evidencian que dichas actividades -al igual que la agraria, la industrial o el desarrollo urbano- son capaces de transformar e incluso crear nuevos paisajes, resultado de la combinación de la naturaleza, la cultura de los agentes que intervienen y la técnica puesta en marcha. Estamos hablando de nuevos paisajes de las energías renovables entendiendo el paisaje como un totalizador histórico, social, económico, ambiental y técnico.

Uno de los mejores exponentes de la dimensión territorial y paisajistica de las energías renovables es el libro Renewable Energies and European Landscapes. Lessons from Southern European Cases (Frolova, Prados y Nadaï, 2015). En este libro ha participado un equipo multidisciplinar, de forma que se analizan cuestiones sociales, políticas y culturales relativas a las relaciones entre energías renovables y paisaje. Los diferentes autores examinan múltiples procesos que acontecen y hacen emerger los nuevos "paisajes de las energías renovables en el sur de Europa". A través de los diferentes recursos energéticos se analiza el alcance de su desarrollo en el paisaje, y se indaga sobre su contribución al establecimiento de nuevos valores del paisaje. En el libro los autores conciben el paisaje de forma amplia, como un proceso material, social, histórico y político, que excede la dimensión estética. El paisaje y el territorio, debidamente anclados en el ámbito local vertebran la obra, debidamente ejemplificados a través de varios estudios de caso, realizados por investigadores de Francia, Italia, Portugal y España. De forma reiterada se plantea en la obra que el objeto de dichos estudios es analizar los paisajes de las energías renovables, integrando ópticas de disciplinas como la historia, la geografía, la sociología y la antropología. Una lectura en clave metodológica de 
la obra, nos ofrece una meritoria panorámica sobre las técnicas más recientes: (i) cualitativas como la Teoría Fundamentada, la Teoría Actor-Red, y otros de carácter antropológico, (ii) cuantitativas y (iii) Sistemas de Información Geográfica. Renewable Energies and European Landscapes (Frolova, Prados y Nadaï, 2015) se estructura en cinco partes que dan cabida a 15 capítulos, que permiten analizar los siguientes temas: (i) conceptualización de los paisajes de las energías renovables, (ii) desarrollo de nuevas energías y paisajes emergentes Frolova et al., 2014), (iii) energía hidroeléctrica y paisajes de montaña, (iv) energías renovables y paisajes protegidos y (v) herramientas de planificación de los paisajes de las energías renovables y su aplicación.

La actividad energética ha estado acompañada por profundas transformaciones paisajísticas y territoriales, pero sólo desde una década éstas ocupan un espacio central en la comunidad científica internacional. Precisamente en la publicación Renewable Energies and European Landscapes, se lleva a cabo un análisis de los paisajes que están actualmente surgiendo con el desarrollo de distintas tecnologías y en diversos contextos -montañas, llanuras y áreas costeras-. Los autores demuestran que el paisaje es tanto una cuestión estética en la planificación espacial de las energías renovables, como un objeto que está profundamente incrustado en las prácticas locales.

\section{CONCLUSIÓN}

La comunidad de geógrafos de forma temprana focalizó sus estudios en los recursos energéticos y los diferentes procesos de transformación hasta su consumo. Este hecho favoreció la emergencia de una dimensión productivista y economicista de la energía como argumento del diferente grado de desarrollo de grandes conjuntos regionales y/o países a escala munidal. Dicha dimensión se prolongó en el tiempo a través de numerosos estudios exploratorios de los principales recursos energéticos que protagonizaban "nuevas alternativas"; desde el carbón, los hidrocarburos o el uranio, hasta las energías renovables. Bajo esta dimensión productivista o económica pocos hablan de relaciones espaciales, de agentes sociales o de impactos que excedan los propios de la extracción de los recursos. Bajo preceptos de la geografía radical surgieron nuevas voces, que abogaban por una geografía de la energía de mayor carácter espacial, social y ambiental. Es el momento de debatir sobre "sistemas energéticos" y la necesidad de una transición hacia un modelo energético menos dependiente de los hidrocarburos.

En la década de 1990 los geógrafos integran la energía como tema transversal en los diferentes estudios. La pujante "Geografía de la energía" de las décadas anteriores acabó diluyéndose entre estudios de la geografía de los transportes o de la geografía del comercio entre otras. Sin embargo, los avances técnicos, el apoyo público y el activo papel del sector privado favorecieron el desarrollo de la energía eólica y fotovoltaica. Las nuevas infraestructuras energéticas presentan una condición paradójica, pues al tiempo que suscitan una amplia aceptación a escala global por los beneficios ambientales, generan rechazo a escala local por el impacto que provocan, cobrando entonces el paisaje una dimensión especial dentro de los estudios geográficos sobre las energías renovables. De especial interés son los trabajos que evalúan las múltiples relaciones entre paisaje, infraestructuras energéticas y percepción ciudadana; y que conducen hacia la hipótesis de la emergencia de nuevos paisajes, los de las energías renovables. 
Apreciamos por lo tanto una dinámica y constante evolución de la investigación sobre la energía desde la disciplina geográfica. Diferenciamos dos tipos de estudios: aquellos que abordan la energía de forma genérica incluyendo todos los recursos, de aquellos que se centran en un recurso o técnica de explotación energética. Los primeros deben su planteamiento sustancialmente al paradigma epistemológico en el que se enmarque la investigación, pues en ellos se observa bien la dimensión con la que se aborda el objeto de estudio. En cambio, si el estudio se centra en un recurso o técnica de explotación, al paradigma epistemológico se le suma el grado de desarrollo del propio recurso objeto de estudio. Así por ejemplo en la última década para el caso del desarrollo eólico y fotovoltaico identificamos desde trabajos de carácter exploratorio, hasta los estudios específicos sobre su integración en el paisaje. En todo caso, independientemente del enfoque, todo estudio elaborado desde la dimensión geográfica ha de partir de una premisa: la relevancia de la energía como transformador y productor social del espacio.

\section{BIBLIOGRAFÍA}

AFONSO, A.I. y MENDES, C. (2015): "Wind power and environmental policies" en Renewable Energies and European Landscapes. Lessons from Southern European Cases (Frolova, M., Prados, M.J., y Nadaï, A. Eds.). Springer Netherlands, 175-191.

ALEXANDER, J.W. y GIBSON, L.J. (1979): Economic geography 2a ed. Englewood Cliffs, Prentice-Hall.

ALLIX, A. (1948). «L'esprit et les méthodes de la géographie ». Les Études Rhodaniennes, vol. 23 (4), 295-310.

ANDRÉS DE, C. y IRANZO, E. (2011): “Desarrollo de las energías renovables y cambios paisajísticos: propuesta de tipología y localización geográfica de los paisajes energéticos de España”, en Energía y territorio: dinámicas y procesos, Alicante, Asociación de Geógrafos Españoles, Colegio de Geógrafos de España y Universidad de Alicante, 97-107.

BARAJA, E. y HERRERO, D. (2010): "Energías renovables y paisaje en Castilla y León: Estudio de caso". Nimbus, $\mathrm{n}^{\circ}$ 25-26, 21-42.

BARAJA, E., HERRERO, D. y PÉREZ-PÉREZ, B. (2015): “A country of windmills: wind energy development and landscape in Spain", en Renewable Energies and European Landscapes. Lessons from Southern European Cases (Frolova, M., Prados, M.J., y Nadaï, A. Eds.). Springer Netherlands, 43-61.

BOUZAROVSKI, S., PASQUALETTI, M.J., y CASTÁN, V. (2017): Routledge research companion to energy geographies. Routledge.

BRIFFAUD, S., HEAULMÉ, E., ANDRÉ-LAMAT, V., DAVASSE, B. y SACAREAU, I. (2015): « The nature of resources: conflicts of landscape in the Pyrenees during the rise of hydroelectric power", en Renewable Energies and European Landscapes. Lessons from Southern European Cases (Frolova, M., Prados, M.J., y Nadaï, A. Eds.). Springer Netherlands, 135-153.

BRÜCHER, W. (1997): « Mehr Energie! Plädoyer für ein vernachlässigtes Objekt der Geographie". Geographische Rundschau, no 49, 330-335.

BRÜCHER, W. (2004): "Geography of Energy”, en International encyclopedia of the social and behavioral sciences (Smelser, N.J. y Baltes, P.B. Eds.). Detroit, Gale-Cengage. 
CALZONETTI, F.J. y SOLOMON, B.D. (1985): Geographical dimensions of energy. Dordrecht, Países Bajos, D. Reidel Pub. Co.

CAPEL, H. (2012): Filosofía y ciencia en la geografía contemporánea: una introducción a la geografía. 2a ed. Barcelona, Ediciones del Serbal.

COOK, E.F. (1976): Man, energy, society. San Francisco, W.H. Freeman.

COWELL, R. (2010): "Wind power, landscape and strategic, spatial planning: The construction of acceptable locations in Wales". Land Use Policy, vol. 27 (2), 222-232.

CURRAN, D.W. (1973) : Géographie mondiale de l'énergie. París, Masson.

CURRAN, D.W. (1981) : La nouvelle donne énergétique. París, Masson.

DESHAIES, M. y HERRERO, D. (2015): "Wind Energy and Natural Parks in European Countries (Spain, France and Germany)", en Renewable Energies and European Landscapes. Lessons from Southern European Cases (Frolova, M., Prados, M.J. y Nadaï, A. Eds.). Springer Netherlands, 217-233.

DEVINE-WRIGHT, P. y DEVINE-WRIGHT, H. (2006): "Social representations of intermittency and the shaping of public support for wind energy in the UK". International Journal of Global Energy Issues, vol. 25 (3/4), 243-256.

DÍAZ-CUEVAS, P. y DOMÍNGUEZ-BRAVO, J. (2015): "GIS, territory and Landscape in renewable energy management in Spain", en Renewable Energies and European Landscapes. Lessons from Southern European Cases (Frolova, M., Prados, M.J. y Nadaï, A. Eds.). Springer Netherlands, 279-294.

DRESCH, J. (1980):“Reflexões sobre a Geografía” en Reflexões sobre a Geografía, São Paulo, Edições da AGB, 5-26.

ESPEJO, C. (2009): "Los biocarburantes en España. Un sector en desarrollo". Boletín de la Asociación de Geógrafos Españoles, $\mathrm{n}^{\circ}$ 50, 111-134.

ESPEJO, C. y GARCÍA, R. (2010): “Agua y energía: producción hidroeléctrica en España”. Investigaciones Geográficas n $\mathrm{n}^{\mathrm{0}}$ 51, 107-129.

ESPEJO, C., MILLÁN, D. y GARCÍA, R. (2016): “Contribución al estudio del sector de los biocarburantes en España”. Scripta Nova, vol. XX, n 548, 15 de noviembre de 2016. Universidad de Barcelona. Disponible en http://www.ub.edu/geocrit/sn/sn-548.pdf

FITZSIMMONS, A.K. y WALTON, K.J. (1978): “Toward a geography of energy: A review of basic sources and literature". Journal of Geography, vol. 77 (2), 61-66.

FROLOVA, M. (2010a): "Landscapes, water policy and the evolution of discourses on hydropower in Spain”. Landscape Research - LANDSC RES, no 35 (2), 235-257.

FROLOVA, M. (2010b): "Los paisajes de la energía eólica: Su percepción social y gestión en España". Nimbus, no 25-26, 93-110.

FROLOVA, M., ESPEJO, C., BARAJA, E. y PRADOS, M.J. (2014): "Paisajes emergentes de las energías renovables en España”. Boletín de la Asociación de Geógrafos Españoles, $n^{\circ} 66,223-252$.

FROLOVA, M., JIMÉNEZ-OLIVENZA, Y., SÁNCHEZ-DEL ÁRBOL, M.-Á., REQUENA A. y PÉREZ-PÉREZ, B. (2015): “The evolution of renewable landscapes in Sierra Nevada (Southern Spain). From small hydro- to a wind-power landscape", en Renewable Energies and European Landscapes. Lessons from Southern European Cases (Frolova, M., Prados, M.J., y Nadaï, A. eds.). Springer Netherlands, 117-134. 
FROLOVA, M., PRADOS, M.J. y NADAÏ, A. (Eds.) (2015): Renewable Energies and European Landscapes: Lessons from Southern European Cases. Springer.

GEORGE, P. (1950): Géographie de l'énergie. París, Librairie de Médicis.

GILBERT, G.K. (1917): Hydraulic-mining debris in the Sierra Nevada. United States Geological Survey. http://pubs.usgs.gov/pp/0105/report.pdf, consultado 25.02.2017.

GUYOL, N.B. (1971): Energy in the perspective of geography. Engle-wood Cliffs, PrenticeHall.

HAGGETT, C. y TOKE, D. (2006): “Crossing the Great Divide - Using Multi-method Analysis to Understand Opposition to Windfarms". Public Administration, vol.84 (1), 103-120.

HAMMARLUND, K., FROLOVA, M. y BRANHULT, A. (2016): “Wind power development and landscape: social participation, opportunities and challenges", en Renewable Energy - Utilisation and System Integration (Wenping Cao y Yihua Hu, Eds.). Intech, $1-16$.

HARE, F.K. (1953): The restless atmosphere. Londres, Hutchinson University Library.

HARE, F.K. (1965): "Energy Exchanges and the General Circulation”. Geography, n50 (3), 229-241.

LINTON, D.L. (1965): “The Geography of Energy”. Geography, vol.50 (3), 197-228.

MANNERS, G. (1964): The geography of energy. Londres, Hutchinson University Library.

MARCUS, M.G. (1978): "Review of Man, Energy, Society". Annals of the Association of American Geographers, vol. 68 (4), 572-574.

MEADOWS, D.H., MEADOWS, D.L., RANDERS, J. y BEHRENS W. (1972): Los Limites del crecimiento: informe al Club de Roma sobre el predicamento de la humanidad. Fondo de Cultura Económica.

MÉRENNE-SCHOUMAKER, B. (2011): Géographie de l'énergie: acteurs, lieux et enjeux. París, Belin.

MICHEL, M. (1978): «L'extraction de l'uranium ». Annales de géographie, nº482, 394-434.

MÖLLER, B. (2006): "Changing wind-power landscapes: regional assessment of visual impact on land use and population in Northern Jutland, Denmark". Applied Energy, $\mathrm{n}^{\circ} 83$ (5), 477-494.

MÖLLER, B. (2010): "Spatial analyses of emerging and fading wind energy landscapes in Denmark". Land Use Policy, vol. 27 (2), 233-241.

NADAÏ, A., KRAUSS, W., ALFONSO, A.I., DRACKLÉ, D., HINKELBEIN, O., LABUSSIÉRE, O., y MENDES, C. (2010): "El paisaje y la transición energética: comparan-do el surgimiento de paisajes de energía eólica en Francia, Alemania y Portugal". Nimbus, $\mathrm{n}^{\circ} 25-26,155-174$.

NADAII, A. y LABUSSIÈRE, O. (2010): "Birds, Wind and the Making of Wind Power Landscapes in Aude, Southern France”. Landscape Research, vol. 35 (2), 209-233.

NYE, D.E. (1990): Electrifying America: social meanings of a new technology, 1880-1940. Cambridge, MIT Press.

ODELL, P. (1963): An economic geography of oil. Londres, Bell.

PASQUALETTI, M.J. (1979): “Energy”. Geographical Survey, nº 8, 35-40.

PASQUALETTI, M.J. (2011): "The geography of energy and the wealth of the world". Annals of the Association of American Geographers, vol. 101 (4), 971-980. 
PIAGET, J., LAZARSFELD, P. y MACKENZIE, W.J.M. (1975): Tendencias de la investigación en ciencias sociales. $2 \mathrm{a}$ ed, Madrid, Alianza.

PRADOS, M.J., BARAJA, E., FROLOVA, M. y ESPEJO, C. (2012): “Integración paisajística y territorial de las energías renovables". Ciudad y territorio: Estudios territoriales, $\mathrm{n}^{\mathrm{o}} 171,127-143$.

PRATT, W.E. y GOOD, P. (1950): World geography of petroleum. Nueva York, Princeton University Press.

SHERLOCK, R.L. (1922): Man as a geological agent; an account of action on inanimate nature. London, H.F. \& G. Witherby

SHESKIN, I.M. (1985): "Petroleum and natural gas", en Geographical dimensions of energy, Dordrecht, D.Reidel Pub. Co., 19-25.

SORRE, M. (1948): Les fondements techniques. Paris, Armand Colin.

THORNTHWITE, C.W. (1961): "The task ahead". Annals of the Association of American Geographers, vol. 51 (4), 345-356.

TOKE, D. BREUKERS, S. y WOLSINK, M. (2008): "Wind power deployment outcomes: How can we account for the differences?" Renewable and Sustainable Energy Reviews, vol. 12 (4), 1129-1147.

TURNER, G.M. (2008): “A comparison of The Limits to Growth with 30 years of reality". Global Environmental Change, vol. 18 (3), 397-411.

VAN DER HORST, D. y TOKE, D. (2010): "Exploring the landscape of wind farm developments; local area characteristics and planning process outcomes in rural England". Land Use Policy, vol. 27 (2), 214-221.

WALKER, G. (1995): "Renewable energy and the public". Land Use Policy, vol. 12 (1), 49-59.

WEST, J., BAILEY, I. y WINTER, M. (2010): "Renewable energy policy and public perceptions of renewable energy: A cultural theory approach”. Energy Policy, vol. 38 (10), 5739-5748.

WILBANKS, T.J. (1985): "Geography and energy: the quest for roles and missions" en Geographical dimensions of energy, Dordrecht, D. Reidel Pub. Co., 497-510.

WOLSINK, M. (2007): "Planning of renewables schemes: Deliberative and fair decisionmaking on landscape issues instead of reproachful accusations of non-cooperation". Energy Policy, vol. 35 (5), 2692-2704.

WOODS, M. (2003): "Conflicting Environmental Visions of the Rural: Windfarm Development in Mid Wales". Sociologia Ruralis, vol. 43 (3), 271-288.

WÜSTENHAGEN, R., WOLSINK, M. y BÜRER, M.J. (2007): "Social acceptance of renewable energy innovation: An introduction to the concept". Energy Policy, vol. 35 (5), 2683-2691.

ZOELLNER, J., SCHWEIZER-RIES, P. y WEMHEUER, C. (2008): « Public acceptance of renewa-ble energies: Results from case studies in Germany". Energy Policy, vol.36 (11), 4136-4141. 